\title{
An obligatory anaerobic Salmonella typhimurium strain redirects M2 macrophages to the M1 phenotype
}

\author{
MEI YANG ${ }^{1-3^{*}}, \mathrm{JUAN}_{\mathrm{XU}}^{2 *}, \mathrm{QI} \mathrm{WANG}^{2 *}, \mathrm{AN}-\mathrm{QIN} \mathrm{ZHANG}^{2}$ and $\mathrm{KUN}^{2} \mathrm{WANG}{ }^{1}$ \\ ${ }^{1}$ Department of Breast Cancer, Cancer Center, Guangdong General Hospital, Guangdong Academy of Medical \\ Sciences, Guangzhou, Guangdong 510080; ${ }^{2}$ Department of Breast Cancer, Guangdong Women and Children's \\ Hospital, Guangzhou, Guangdong 511400; ${ }^{3}$ Department of General Surgery, General Hospital of Guangzhou \\ Military Command of People's Liberation Army, Guangzhou, Guangdong 510010, P.R. China
}

Received July 19, 2016; Accepted November 20, 2017

DOI: $10.3892 / \mathrm{ol} .2018 .7742$

\begin{abstract}
A genetically engineered Salmonella typhimurium strain that may be applied in the medically useful therapeutic strategy of using bacterial agents to target breast cancer in a tumor-bearing nude mouse model has been previously reported. Furthermore, immune cell accumulation in breast tumor types has been observed, particularly distributed in regions surrounding the bacteria. M2 macrophages are associated with breast cancer aggressiveness, whereas M1 macrophages are prone to devouring bacteria and killing cancer cells. Therefore, this engineered tumor-targeting salmonella strain was used in an attempt to reverse the phenotype of M2 macrophages into the M1 phenotype. Subsequent to the co-culture of M2 macrophages with the bacteria for a short time, $>50 \%$ of the M2 macrophages were invaded by bacteria. These M2 macrophages exhibited a decreased expression of mannose receptor (an M2 phenotypic marker) and increased expression of human leukocyte antigen-antigen $\mathrm{D}$ related (an M1 phenotypic marker). The results of the present study indicated that differentiated M2 macrophages may be redirected into the M1 phenotype following exposure to the engineered bacteria stimulus. This effect may be a potential mechanism by which bacteria retard tumor growth. Thus, this engineered bacterium may be a useful candidate for targeting and redirecting M2 macrophages into the M1 phenotype.
\end{abstract}

Correspondence to: Dr Mei Yang or Dr Kun Wang, Department of Breast Cancer, Cancer Center, Guangdong General Hospital, Guangdong Academy of Medical Sciences, 106 Zhongshan 2nd Road, Guangzhou, Guangdong 510080, P.R. China

E-mail: bayberry513@yahoo.com

E-mail: gzwangkun@126.com

*Contributed equally

Key words: engineered bacteria, breast cancer, macrophage, tumor-targeting therapy

\section{Introduction}

The potential use of bacteria for cancer treatment has been extensively investigated in previous years. Bacteria, including Bifidobacterium (1,2), Clostridium (3) and Salmonella have been demonstrated to preferentially target and replicate in the hypoxic and necrotic regions of a tumor, resulting in tumor repression (4-7). In a previous study, a synthetic biology approach was used to generate the novel Salmonella typhimurium strain YB1 (YB1) (8). This bacterium specifically colonizes and proliferates in the hypoxic/necrotic areas of the tumor, but avoids normal organs and retards tumor growth (8). Furthermore, a previous study reported that numerous macrophages accumulate in breast tumors and are associated with a poor prognosis (9).

Macrophages are heterogeneous cells that respond differently to various stimulating signals and display numerous different phenotypes (5). The M1 and M2 macrophage phenotypes represent the two extremes of a broad range of macrophage functional states. Fully polarized M1 (or classically activated) macrophages are stimulated by microbial agents or pro-inflammatory factors, including lipopolysaccharide (LPS), whereas M2 (or alternatively activated) macrophages respond to anti-inflammatory molecules, including interleukin-4 (IL-4) $(10,11)$. Macrophages located in the stroma of breast cancer tissues [known as tumor-associated macrophages (TAMs)] are primarily M2 macrophages activated by IL-4-producing cluster of differentiation (CD)4+ T cells (12). TAMs are the most notable migratory hematopoietic cell type in the tumor microenvironment and promote the invasiveness of breast cancer cells (13).

Clinically, a large amount of macrophage infiltration in tumor sections from patients with breast cancer has been observed using CD68 immunohistochemical staining. TAMs are associated with breast cancer aggressiveness and promote cancer metastasis, whereas M1 macrophages are prone to killing cancer cells and devouring bacteria (14). Furthermore, studies have revealed that TAMs (which are primarily M2 macrophages activated by IL-4) exhibit a CD206 high/human leukocyte antigen-antigen D related (HLA-DR) ${ }^{\text {low }}$ phenotype that is associated with immune suppression (15-17). Therefore, CD206 and HLA-DR may be used as markers for M1 and 
M2 macrophage phenotype analysis (15). In the present study, the newly engineered tumor-targeting YB1 strain was used in order to attempt to redirect M2 macrophages into the M1 phenotype. More than half of the M2 macrophages devoured the bacteria after $2 \mathrm{~h}$ of co-culture. These M2 macrophages exhibited a decreased CD206 expression and an increased HLA-DR expression. Therefore, the IL-4-activated M2 macrophages switched from the CD206 ${ }^{\text {high }} / \mathrm{HLA}-\mathrm{DR}{ }^{\text {low }}$ phenotype to the CD206 ${ }^{\text {low }} / \mathrm{HLA}-\mathrm{DR}{ }^{\text {high }}$ phenotype subsequent to co-culture with the engineered YB1 strain. The present study indicates that differentiated M2 macrophages may be redirected into an M1 phenotype following exposure to different stimuli. This finding may reflect a potential mechanism by which bacteria retard tumor growth. Therefore, these engineered bacteria may be used as a vector to target tumors.

\section{Materials and methods}

Patient samples and macrophage immunohistochemistry staining. All tumor samples from breast-infiltrating ductal carcinomas were obtained from female patients (mean age, 45 years; age range, 35-55 years) at the Guangdong Women and Children's Hospital (Guangdong, China). The samples were used with written informed consent and ethical approval was obtained from the Internal Review and the Ethics Boards of Guangdong Women and Children's Hospital (Guangdong, China).

The samples were fixed in $10 \%$ formalin for $>2 \mathrm{~h}$ at room temperature, paraffin-embedded $\left(3 \mathrm{~min}\right.$ at $\left.56^{\circ} \mathrm{C}\right)$ and sectioned into $5 \mu \mathrm{M}$-thick slices. The macrophages were visualized by immunohistochemistry staining using an anti-CD68 antibody (cat. no. M0814; dilution, 1:200; Dako; Agilent Technologies, Inc., Santa Clara, CA, USA), and sections were treated using this antibody overnight at $4^{\circ} \mathrm{C}$. For details, please refer to reference (18).

Bacterial culture. The bacterial YB1 strain was cultured in lysogeny broth medium overnight (12 to $16 \mathrm{~h}$ ) (Sigma-Aldrich; Merck KGaA, Darmstadt, Germany) supplemented with chloramphenicol and 2,3-diaminopropionic acid (Sigma-Aldrich; Merck KGaA) at $37^{\circ} \mathrm{C}(8)$.

Isolation and activation of human monocyte-derived macrophages. Institutional ethical approval was obtained from the Internal Review and the Ethics Boards of Guangdong Women and Children's Hospital, Guangdong, China prior to conducting the study. Human mononuclear cells were isolated from $100 \mathrm{ml}$ peripheral blood of healthy donors by Ficoll density gradient centrifugation $\left(20^{\circ} \mathrm{C}\right.$ at $250 \times \mathrm{g}$ for $\left.20 \mathrm{~min}\right)$, as previously described (18). The resulting monocyte-derived macrophages were activated by the addition of IL-4 (45 ng/ml) to the culture medium for 3 days (19), and LPS (20 ng/ml) was added as a control.

Bacteria and macrophage co-culture. Isolated macrophages were activated by IL-4. The bacterial YB1 strain, which carried the green fluorescent protein (GFP)-tagged plasmid, was added to the macrophage culture for a final bacterial concentration of $5 \times 10^{6} / \mathrm{ml}$. The cultures were incubated at $37^{\circ} \mathrm{C}$ under hypoxic conditions as previously described (8) for $2 \mathrm{~h}$.

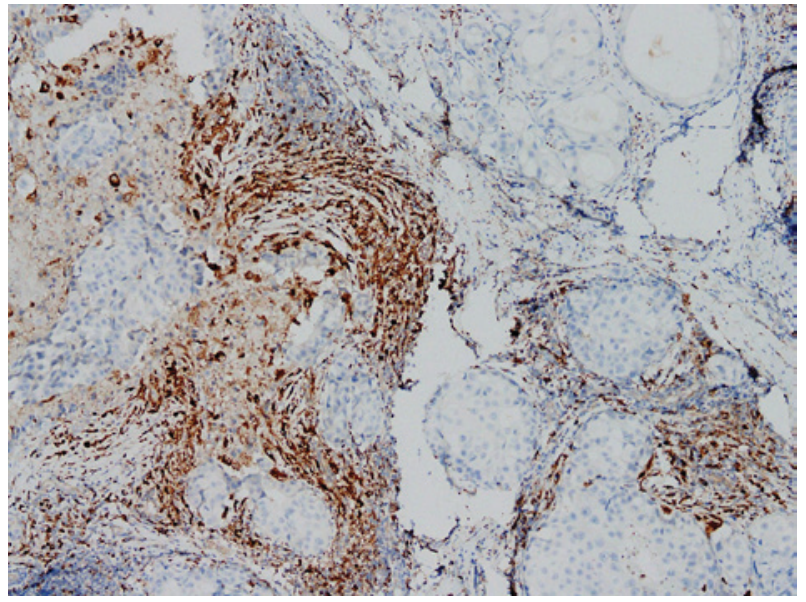

Figure 1. Immunohistochemical staining of breast cancer tissue revealing the mannose receptor-positive macrophages present (magnification, x100).

Then, the bacteria were washed away and the macrophages were harvested for further analysis.

Flow cytometry. Following the co-culture, the macrophages were fixed in paraformaldehyde $(4 \%)$ at $4^{\circ} \mathrm{C}$ for $0.5-2 \mathrm{~h}$. The macrophage phagocytic rate of YB1 (GFP) was detected using flow cytometry. CD206 or HLA-DR expression levels in the macrophages were also determined using flow cytometry. Briefly, the macrophages $\left(10^{5}-10^{6} / \mathrm{ml}\right)$ were collected, fixed in $4 \%$ paraformaldehyde at $4^{\circ} \mathrm{C}$ for $0.5-2 \mathrm{~h}$, blocked with $5 \%$ bovine serum albumin for $20 \mathrm{~min}$ at room temperature and stained using a phycoerythrin-conjugated CD206 (dilution, 1:20; cat no. 321105; BioLegend, Inc., San Diego, CA, USA) or allophycocyanin-conjugated HLA-DR (dilution, 1:20; cat no. 307609; BioLegend, Inc.) antibody for $1 \mathrm{~h}$ at $4^{\circ} \mathrm{C}$. The corresponding isotype control was included in each test. Then, CD206 or HLA-DR expression was analyzed using flow cytometry. FlowJo software (version 7.6.1; FlowJo LLC, Ashland, OR, USA) was used to analyze the data.

Western blotting. Cells were lysed using radioimmunoprecipitation assay lysis buffer $(50 \mathrm{mM}$ TrisHCl pH 7.4, $150 \mathrm{mM}$ $\mathrm{NaCl}, 2 \mathrm{mM}$ EDTA, 1\% NP-40, 0.1\% SDS) and protease inhibitors (Sigma-Aldrich; Merck KGaA). Cells were lysed with lysis buffer for $15-20 \mathrm{~min}$ at $4^{\circ} \mathrm{C}$. HLA-DR expression was determined according to a previously described protocol (18). The proteins (20 $\mu$ l per lane) were separated on $10 \%$ SDS-PAGE gels and transferred onto nitrocellulose membranes. The membranes were blocked in 5\% non-fat milk for $2 \mathrm{~h}$ at room temperature and then incubated with antibodies against HLA-DR (1:5,000; rabbit monoclonal IgG antibody; cat no. ab92511; Abcam, Cambridge, UK) overnight at $4^{\circ} \mathrm{C}$. Subsequent to washing using $0.1 \%$ TBST $(50 \mathrm{mM}$ tris, $150 \mathrm{mM} \mathrm{NaCl}, 0.1 \%$ Tween $20, \mathrm{pH} 7.4$ ) three times (10 min each), the membrane was incubated with a horseradish peroxidase (HRP)-conjugated secondary antibody (GE Healthcare, Chicago, IL, USA) for $1 \mathrm{~h}$ at room temperature. Then, the membranes were washed again using $0.1 \%$ TBST three times (10 min each). The HRP signal was visualized using enhanced chemiluminescence (ChemiDoc MP Imaging System; 

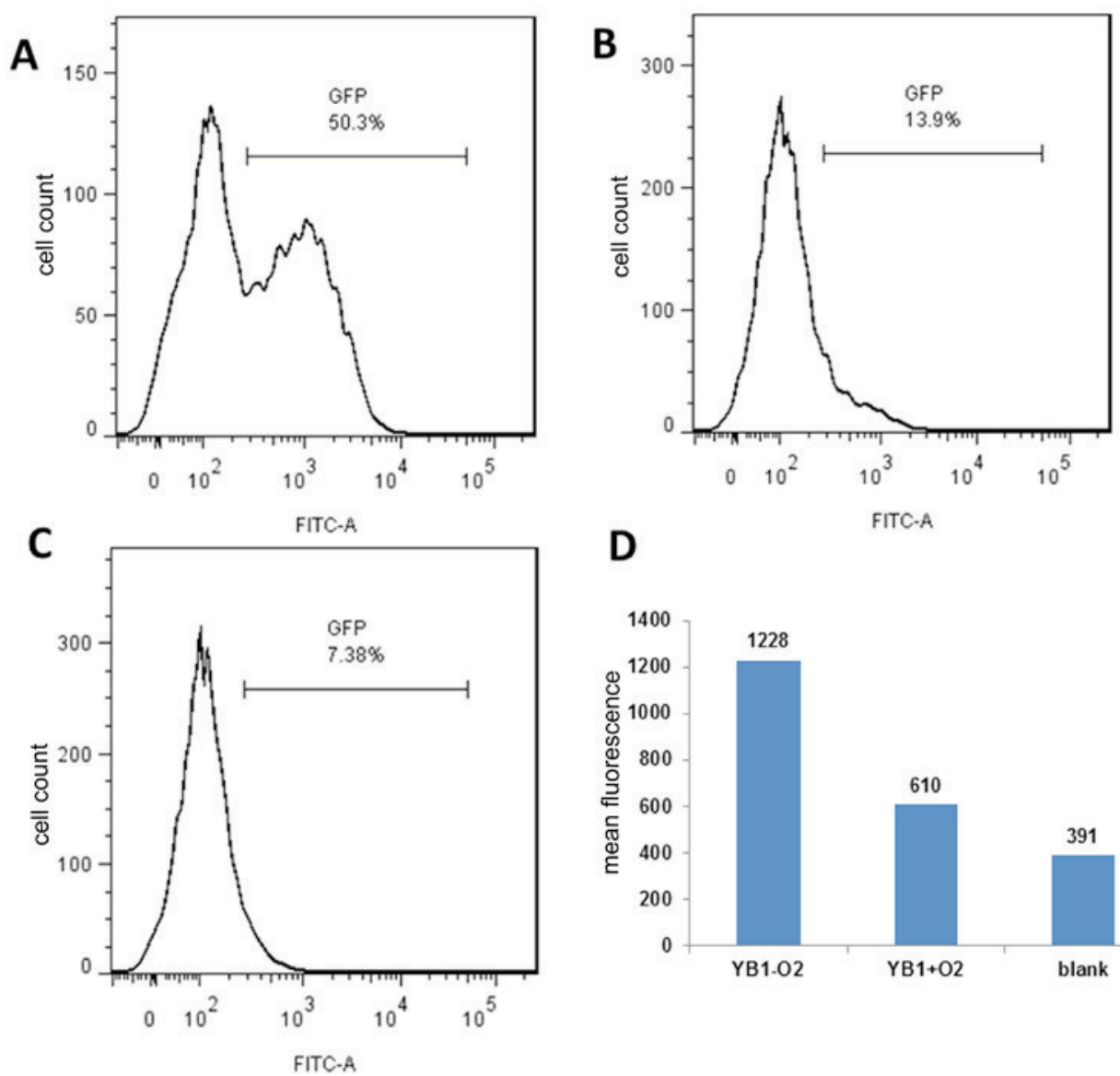

D

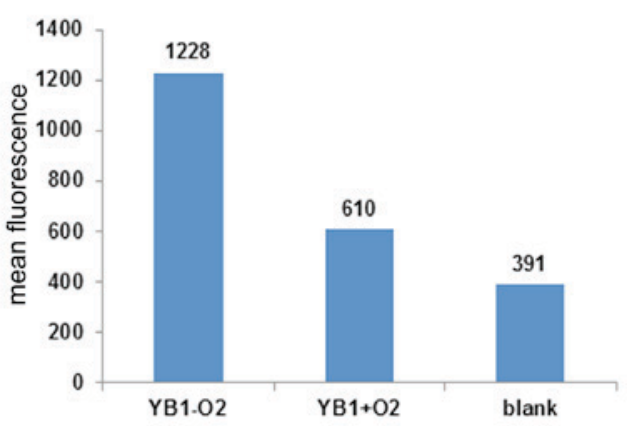

Figure 2. YB1 strain invasion rate between hypoxic and normal conditions, determined by flow cytometry analysis. YB1 invasion rate under (A) hypoxic and (B) normal conditions, with (C) demonstrating the results of the blank control. (D) Mean fluorescence between hypoxic and normal conditions. YB1- ${ }_{2}$ : under hypoxic conditions; $\mathrm{YB} 1+\mathrm{O}_{2}$ : under normal conditions. GFP, green fluorescent protein; FITC-A, fluorescein isothiocyanate; YB1, Salmonella typhimurium strain YB1.

Bio-Rad Laboratories, Inc., Hercules, CA, USA), and analyzed using Image Lab (version 5.2.1; Bio-Rad Laboratories, Inc.).

\section{Results}

Macrophage infiltration in breast tumor tissues. The engineered YB1 strain may specifically target and survive in a solid tumor. To confirm macrophage infiltration in the breast tumor, clinical breast cancer samples were collected and CD68 immunohistochemistry staining was used to demonstrate the macrophage distribution. As presented in Fig. 1, a large number of macrophages (CD68-positive) infiltrated the breast tumor, particularly in the tumor-associated stromal border, a result consistent with previous reports (12). This result implies that bacteria may be able to target macrophages localized in the breast tumor.

YB1 invaded M2 macrophages under hypoxic conditions. The engineered YB1 strain only survives under hypoxic conditions (8). However, whether YB1 may invade M2 macrophages under hypoxic conditions remains unknown. Thus, an anaerobic microenvironment was stimulated in vitro and co-cultured M2 macrophages with the YB1 strain with the GFP-tagged plasmid for $2 \mathrm{~h}$. Flow cytometry analysis was used to determine the YB1 invasion rate. As presented in Fig. 2A, YB1 had a high invasion rate under hypoxic conditions; after $2 \mathrm{~h},>50 \%$ of the macrophages were invaded. In contrast, the invasion rate was very low under normal conditions (21\% oxygen) (Fig. 2B) as YB1 may not survive. Fig. $2 \mathrm{C}$ presented the results of the blank control. It was additionally identified that the mean fluorescence was substantially higher under hypoxic conditions than under normal conditions, indicating that $>1$ bacterium invaded each macrophage (Fig. 2D).

M2 macrophages exhibited increased HLA-DR and decreased CD206 expression. Subsequent to co-culturing M2 macrophages with YB1, the macrophages were collected and flow cytometry was used to determine the HLA-DR expression levels (Fig. 3A). LPS activated the macrophages to express higher levels of HLA-DR compared with those in the IL-4-activated macrophages. Notably, the YB1 alone strain induced the highest HLA-DR expression in macrophages, suggesting the high efficiency of YB1 in activating the M1 macrophage phenotype. When the macrophages were activated in advance with IL-4 and then co-cultured with YB1, an increase in HLA-DR expression was observed compared with LPS or IL-4 alone activated macrophages. Western blotting confirmed these results (Fig. 3B). Next, the M2 macrophage phenotype marker CD206 was examined (Fig. 3C) and it was identified that IL-4-activated macrophages expressed higher CD206 levels compared with LPS-activated macrophages. The YB1 strain alone reduced the CD206 expression levels compared with 
A

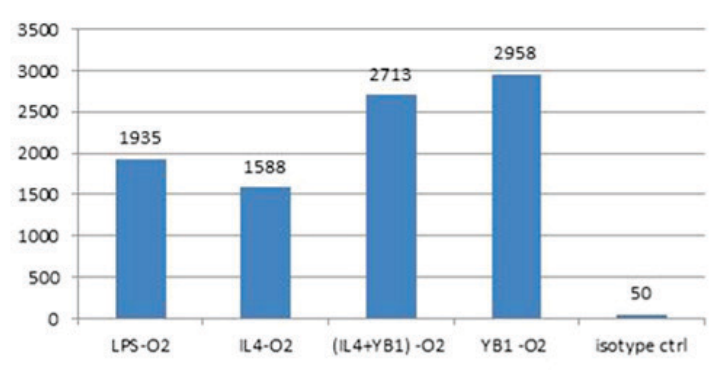

B

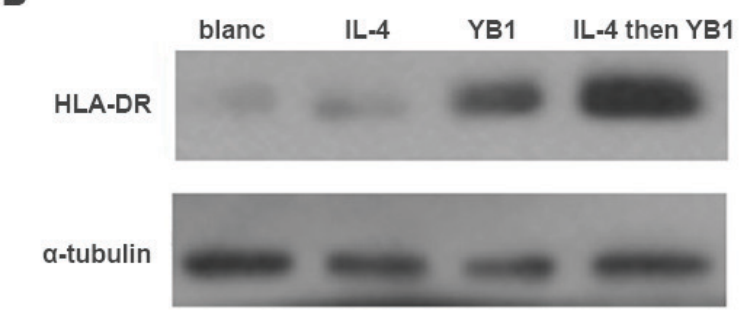

C

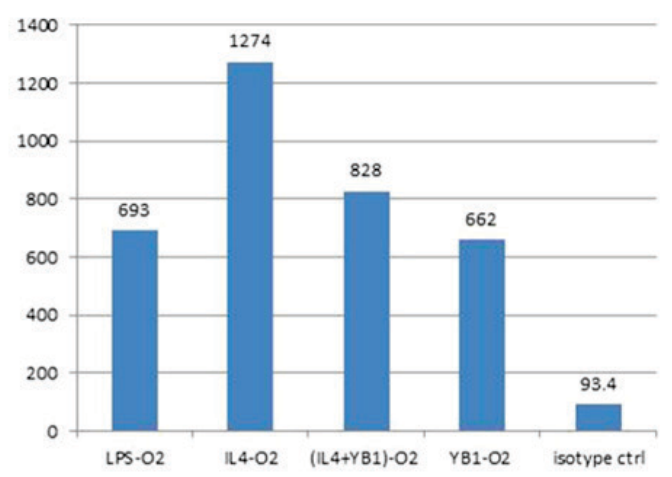

Figure 3. M2 macrophages exhibited increased HLA-DR and decreased CD206 expression levels. (A) HLA-DR expression levels in M2 macrophages activated using LPS, IL-4, YB1, a combination of YB1 and IL-4 or an isotype. (B) HLA-DR expression confirmed using western blotting. (C) CD206 expression levels in M2 macrophages activated using LPS, IL-4, YB1, a combination of YB1 and IL-4 or an isotype. HLA-DR, human leukocyte antigen-antigen D related; CD206, mannose receptor; LPS, lipopolysaccharide; IL, interleukin; YB1, Salmonella typhimurium strain YB1.

every other group. Notably, the YB1 strain reduced CD206 expression in the IL-4-activated M2 macrophages.

\section{Discussion}

Although a broad range of macrophage subsets have been identified, the two major macrophage populations are the M1 (classically activated) macrophages and M2 (alternatively activated) macrophages (20). These two different types of macrophages have different effects on tumor progression. As early as 1980, studies have demonstrated that bacterial LPS activates macrophages (21-23) to specifically kill tumor cells including breast cancer cells but has no effect on normal cells $(24,25)$. This effect on macrophages requires LPS for maintenance (26). Additionally, macrophages themselves possess a phagocytic ability. When a tumor occurs, macrophages migrate to the tumor location, as directed by the action of chemokines, and devour the tumor cells (27). Macrophages may kill tumor cells, though it remains unknown why this killing effect halts tumor growth and distant metastasis (28). Beyond the tumor immune escape mechanism (29), studies have identified that macrophages are induced by tumor cells in the tumor microenvironment and develop tumor-promoting properties (M2 type, otherwise known as TAMs) $(30,31)$. Statistical data analyses have revealed that the proportion of TAMs in solid tumor tissues may be as high as $80 \%$ (29). Likewise, numerous macrophages have been detected in clinical breast cancer samples and, furthermore, macrophage infiltration and breast cancer metastasis are associated (32-34). However, although M1 and M2 macrophages serve different functions in tumor progression, there is no absolute boundary between the two types of macrophages. In the tumor microenvironment, factors including the MHC expression level in tumor cells and the oxygen pressure in the microenvironment affect the macrophage phenotype (29). Therefore, the phenotype of differentiated macrophages may change. This finding indicates a novel target of tumor treatment: Macrophages in the tumor microenvironment. If M2 macrophages may be directed to become the M1 type, one of the drivers of tumor progression would be eliminated, and result in the gain of one more helper to kill tumor cells.

Deng et al (35) has demonstrated that suppression of heme oxygenase- 1 in TAMs in a breast cancer mouse model alternatively activates the switching of the M2 macrophage type to the classically activated M1 macrophage type. However, the 'weapons' used to specifically target macrophages in breast cancer are still lacking.

In the present study, a novel engineered Salmonella strain (YB1) was reported to induce increased HLA-DR expression and decreased CD206 expression in differentiated M2 macrophages. These M2 macrophages changed from the CD206 ${ }^{\text {high }} / \mathrm{HLA}-\mathrm{DR}{ }^{\text {low }}$ phenotype to the CD206 ${ }^{\text {low }} / \mathrm{HLA}-\mathrm{DR}^{\text {high }}$ phenotype, indicating an M2 to M1-type switch. This result suggests a potential use for the engineered tumor-targeting bacteria YB1 in redirecting M2 type macrophages into the M1 type and thus suppressing tumor growth.

Overall, the results suggest that the engineered bacterial YB1 strain may be a good candidate for targeting and redirecting M2 macrophages into the M1 type. In addition to its tumor targeting ability, these bacteria may survive and proliferate in the tumor microenvironment; therefore, the effects would be long-lasting, and the activation of the M1 type would be sustained. Furthermore, for safety, this engineered Salmonella YB1 strain is controllable and may be eliminated by antibiotics. Finally, the genetic background of YB1 is clear and may be engineered to carry further 'weapons' in order to kill cancer cells. 


\section{Acknowledgements}

The present study was supported by the National Natural Science Foundation of China (grant no. 81202076) and the Guangzhou Science and Technology Program (grant no. 2014J2200007). The authors would like to thank Dr Jian-dong Huang (Hong Kong University) for supplying the bacteria strain.

\section{References}

1. Yazawa K, Fujimori M, Nakamura T, Sasaki T, Amano J, Kano Y and Taniguchi S: Bifidobacterium longum as a delivery system for gene therapy of chemically induced rat mammary tumors. Breast Cancer Res Treat 66: 165-170, 2001.

2. Fujimori M: Anaerobic bacteria as a gene delivery system for breast cancer therapy. Nihon Rinsho 66: 1211-1218, 2008 (In Japanese).

3. Liu SC, Ahn GO, Kioi M, Dorie MJ, Patterson AV and Brown JM: Optimized clostridium-directed enzyme prodrug therapy improves the antitumor activity of the novel DNA cross-linking agent PR-104. Cancer Res 68: 7995-8003, 2008.

4. Zhao M, Yang M, Ma H, Li X, Tan X, Li S, Yang Z and Hoffman RM: Targeted therapy with a Salmonella typhimurium leucine-arginine auxotroph cures orthotopic human breast tumors in nude mice. Cancer Res 66: 7647-7652, 2006.

5. Low KB, Ittensohn M, Le T, Platt J, Sodi S, Amoss M, Ash O, Carmichael E, Chakraborty A, Fischer J, et al: Lipid A mutant Salmonella with suppressed virulence and TNFalpha induction retain tumor-targeting in vivo. Nat Biotechnol 17: 37-41, 1999.

6. Felgner S, Kocijancic D, Frahm M, Curtiss R III, Erhardt M and Weiss S: Optimizing salmonella enterica serovar typhimurium for bacteria-mediated tumor therapy. Gut microbes 7: 171-177, 2016.

7. Frahm M, Felgner S, Kocijancic D, Rohde M, Hensel M, Curtiss R III, Erhardt M and Weiss S: Efficiency of conditionally attenuated Salmonella enterica serovar Typhimurium in bacterium-mediated tumor therapy. mBio 6: pii: e00254-15, 2015.

8. Yu B, Yang M, Shi L, Yao Y, Jiang Q, Li X, Tang LH, Zheng BJ, Yuen KY, Smith DK, et al: Explicit hypoxia targeting with tumor suppression by creating an 'obligate' anaerobic Salmonella Typhimurium strain. Sci Rep 2: 436, 2012.

9. Tang X: Tumor-associated macrophages as potential diagnostic and prognostic biomarkers in breast cancer. Cancer Lett 332: $3-10,2013$

10. Luo Y, Zhou H, Krueger J, Kaplan C, Lee SH, Dolman C, Markowitz D, Wu W, Liu C, Reisfeld RA and Xiang R: Targeting tumor-associated macrophages as a novel strategy against breast cancer. J Clin Invest 116: 2132-2141, 2006.

11. Leek RD, Lewis CE, Whitehouse R, Greenall M, Clarke J and Harris AL: Association of macrophage infiltration with angiogenesis and prognosis in invasive breast carcinoma. Cancer Res 56: 4625-4629, 1996

12. DeNardo DG, Barreto JB, Andreu P, Vasquez L, Tawfik D, Kolhatkar N and Coussens LM: CD4(+) T cells regulate pulmonary metastasis of mammary carcinomas by enhancing protumor properties of macrophages. Cancer cell 16: 91-102, 2009.

13. Zhang C, Gao L, Cai Y, Liu H, Gao D, Lai J, Jia B, Wang F and Liu Z: Inhibition of tumor growth and metastasis by photoimmunotherapy targeting tumor-associated macrophage in a sorafenib-resistant tumor model. Biomaterials 84: 1-12, 2016.

14. Khabbazi S, Goumon Y and Parat MO: Morphine modulates interleukin-4- or breast cancer cell-induced pro-metastatic activation of macrophages. Sci Rep 5: 11389, 2015.

15. Su S, Liu Q, Chen J, Chen J, Chen F, He C, Huang D, Wu W, Lin L, Huang W, et al: A positive feedback loop between mesenchymal-like cancer cells and macrophages is essential to breast cancer metastasis. Cancer cell 25: 605-620, 2014.
16. Dangaj D, Abbott KL, Mookerjee A, Zhao A, Kirby PS, Sandaltzopoulos R, Powell DJ Jr, Lamazière A, Siegel DL, Wolf C and Scholler N: Mannose receptor (MR) engagement by mesothelin GPI anchor polarizes tumor-associated macrophages and is blocked by anti-MR human recombinant antibody. PLoS One 6: e28386, 2011.

17. Kuang DM, Wu Y, Chen N, Cheng J, Zhuang SM and Zheng L: Tumor-derived hyaluronan induces formation of immunosuppressive macrophages through transient early activation of monocytes. Blood 110: 587-595, 2007.

18. Yang M, Chen J, Su F, Yu B, Su F, Lin L, Liu Y, Huang JD and Song E: Microvesicles secreted by macrophages shuttle invasion-potentiating microRNAs into breast cancer cells. Mol cancer 10: 117, 2011.

19. Chen J, Yao Y, Gong C, Yu F, Su S, Chen J, Liu B, Deng H, Wang F, Lin L, et al: CCL18 from tumor-associated macrophages promotes breast cancer metastasis via PITPNM3. Cancer Cell 19: 541-555, 2011.

20. Castoldi A, Naffah de Souza C, Câmara NO and Moraes-Vieira PM: The macrophage switch in obesity development. Front Immunol 6: 637, 2016.

21. Hamaidia M, Staumont B, Duysinx B, Louis R and Willems L: Improvement of malignant pleural mesothelioma immunotherapy by epigenetic modulators. Curr Top Med Chem 16: 777-787, 2016.

22. Murray PJ and Wynn TA: Protective and pathogenic functions of macrophage subsets. Nat Rev Immunol 11: 723-737, 2011.

23. Hambleton J, Weinstein SL, Lem L and DeFranco AL: Activation of c-Jun N-terminal kinase in bacterial lipopolysaccharide-stimulated macrophages. Proc Natl Acad Sci USA 93: 2774-2778, 1996.

24. Chimal-Ramírez GK, Espinoza-Sánchez NA, Chávez-Sánchez L, Arriaga-Pizano L and Fuentes-Pananá EM: Monocyte differentiation towards protumor activity does not correlate with M1 or M2 phenotypes. J Immunol Res 2016: 6031486, 2016.

25. Guo H, Liu Y, Gu J, Wang Y, Liu L, Zhang P and Li Y: Endostatin inhibits the growth and migration of 4T1 mouse breast cancer cells by skewing macrophage polarity toward the M1 phenotype. Cancer Immunol Immunother 65: 677-688, 2016.

26. Cameron DJ and Churchill WH: Cytotoxicity of human macrophages for tumor cells: Enhancement by bacterial lipopolysaccharides (LPS). J Immunol 124: 708-712, 1980.

27. Sinha P, Clements VK, Miller S and Ostrand-Rosenberg S: Tumor immunity: A balancing act between $\mathrm{T}$ cell activation, macrophage activation and tumor-induced immune suppression. Cancer Immunol Immunother 54: 1137-1142, 2005.

28. Mills CD, Lenz LL and Harris RA: A breakthrough: Macrophage-directed cancer immunotherapy. Cancer Res 76: 513-516, 2016.

29. Mitra R, Singh S and Khar A: Antitumour immune responses. Expert Rev Mol Med 5: 1-19, 2003

30. Mantovani A, Sozzani S, Locati M, Allavena P and Sica A: Macrophage polarization: Tumor-associated macrophages as a paradigm for polarized M2 mononuclear phagocytes. Trends Immunol 23: 549-555, 2002.

31. Wahl LM and Kleinman HK: Tumor-associated macrophages as targets for cancer therapy. J Natl Cancer Inst 90: 1583-1584, 1998.

32. Ueno T, Toi M, Saji H, Muta M, Bando H, Kuroi K, Koike M, Inadera $\mathrm{H}$ and Matsushima K: Significance of macrophage chemoattractant protein-1 in macrophage recruitment, angiogenesis, and survival in human breast cancer. Clin Cancer Res 6: 3282-3289, 2000.

33. Leek RD and Harris AL: Tumor-associated macrophages in breast cancer. J Mammary Gland Biol Neoplasia 7: 177-189, 2002.

34. Valković T, Dobrila F, Melato M, Sasso F, Rizzardi C and Jonjić N: Correlation between vascular endothelial growth factor, angiogenesis, and tumor-associated macrophages in invasive ductal breast carcinoma. Virchows Arch 440: 583-588, 2002.

35. Deng R, Wang SM, Yin T, Ye TH, Shen GB, Li L, Zhao JY, Sang YX, Duan XG and Wei YQ: Inhibition of tumor growth and alteration of associated macrophage cell type by an HO-1 inhibitor in breast carcinoma-bearing mice. Oncol Res 20: 473-482, 2013. 